\title{
EIT Image Reconstruction by Modified Data
}

\author{
B. Gong, B. Schullcke, S. Krueger-Ziolek, K. Moeller \\ Institute of Technical Medicine, Furtwangen University, Villingen-Schwenningen, Germany \\ Email: bo.gong@hs-furtwangen.de
}

How to cite this paper: Gong, B., Schullcke, B., Krueger-Ziolek, S. and Moeller, K. (2016) EIT Image Reconstruction by Modified Data. J. Biomedical Science and Engineering, 9, 99-106.

http://dx.doi.org/10.4236/jbise.2016.910B013

Received: July 26, 2016

Accepted: September 20, 2016

Published: September 23, 2016

\begin{abstract}
Electrical impedance tomography (EIT) is a radiation-free imaging method. Canonically, in lung EIT, 16 electrodes are placed horizontally on the thorax skin. By injecting currents through electrodes attached to the skin, a set of induced voltage measurements can be collected. The conductivity distribution on the chest plane can be obtained from these electrical boundary conditions. It has been reported that the adjacent current injection pattern is sub-optimal for EIT reconstruction. However, this adjacent current injection pattern is commonly used in commercially available EIT devices. In this study, we modify the boundary conditions according to the superposition principle of the electrical field. As a result, boundary conditions of the adjacent current pattern will be transformed to those corresponding to "skip-3" current injection pattern. Simulation results indicated that reconstruction benefits from the modified boundary conditions.
\end{abstract}

\section{Keywords}

Electrical Impedance Tomography, Current Pattern, Superposition Principle

\section{Introduction}

Electrical Impedance Tomography (EIT) is a radiation-free imaging method. It attempts to reveal the conductivity distribution changes inside the human body of two time instants through electrical data obtained via the electrodes attached to the boundary. In lung EIT, commonly 16 electrodes are placed equidistantly on the boundary of a horizontal chest plane.

We denote the conductivity of the domain changes between two time steps by $\Delta s$ and the measured voltage changes on the electrodes by a vector $\Delta V$. Under the FEM framework with $M$ elements, the conductivity change $\Delta s$ is represented by a $M \times 1$ vector. Approximately, there exists the following relation:

$$
J \Delta s \approx \Delta V
$$


where $J$ denotes the Jacobian matrix calculated at the constant conductivity 1 :

$$
J_{i j}=\left.\frac{\Delta V_{i}}{\Delta s_{j}}\right|_{1}
$$

Jacobian matrix is commonly calculated by studying the first order perturbation of conductivity on each element [1]. Briefly, under FEM framework, the potential distribution can be solved by forward model. The perturbations on each element can be determined by the simulated potential information [1].

In commercially obtained EIT devices (PulmoVista 500, Dräger Medical, Lübeck, Germany), electrical currents are injected through the electrodes adjacently (Figure 1(a)). Other current injection patterns have been studied by A. Andy et al. in simulation [2]. The authors compared several current injection patterns based on a predefined distinguish ability parameter. They concluded that the adjacent current injection patterns are suboptimal in distinguish ability, especially in the inner-most part of the domain. The authors further suggested injecting currents through electrodes by skipping several electrodes in between (Figure 1(b)). Incited by this result, we propose a framework that reforms the measurement data that are collected under adjacent current injection pattern by the superposition principle of the electrical field.

\section{Method}

\subsection{Superposition Principle}

Electric fields satisfy the superposition principle. This is because Maxwell's equations are linear. In this study, we apply this principle to modify the boundary conditions induced by the adjacent injection pattern (Figure 1(a)). For simplicity, we assume $\left(e_{i}, e_{j}\right)$ and $\left(e_{j}, e_{k}\right)$ to be neighboring electrode pairs in a 16-electrode EIT system.

Suppose an alternative electrical current of $5 \mathrm{~mA}$ is injected into two electrodes $\left(e_{i}, e_{j}\right)$. Thereby, an electric field $E_{i j}$ will be generated. The induced voltage $V_{i j}$ on two electrodes $\left(e_{i}^{\prime}, e_{j}^{\prime}\right)$ can be measured. Similarly, if the same amount of current is injected into electrodes $\left(e_{j}, e_{k}\right)$, the corresponding electrical field $E_{j k}$ and the voltage $V_{j k}$ on electrodes $\left(e_{i}^{\prime}, e_{j}^{\prime}\right)$ can be determined. In addition, if the current is injected through electrodes $\left(e_{j}, e_{k}\right)$, a new of electric field and set of voltage measurement on $\left(e_{i}^{\prime}, e_{j}^{\prime}\right)$, denoted by $E_{i k}$ and $V_{i k}$ can be obtained. It follows from the superposition principle that:

$$
E_{i k}=E_{i j}+E_{j k} .
$$

Hence, the induced voltages between electrodes $e_{i}^{\prime}$ and $e_{j}^{\prime}$ satisfy the following relation:

$$
V_{i k}=V_{i j}+V_{j k} .
$$

That is, the boundary conditions in the EIT problem can be linearly combined. Suppose the original measurement data are collected under adjacent injection pattern. It follows that the voltage measurements of "skip-n" injection pattern can be hypothetically obtained. In this paper, we use always the adjacent voltage measurement pattern. 
(a)

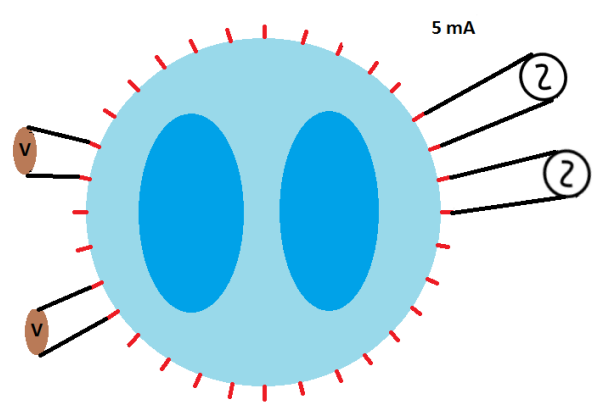

(b)

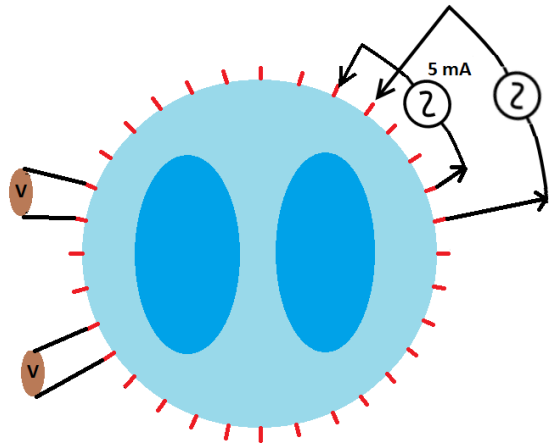

Figure 1. Different current patterns. (a) Demonstrates the adjacent current injection pattern. The so called "skip-3" current injection pattern is shown in (b). Note that for both current injection patterns, the measurement pattern is fixed to be adjacent in this study.

In the real world, all measurements are contaminated by noises. The measurement noise in EIT is commonly assumed to be independent between each electrode pair [2]. For simplicity, we assume an additive white noise on the voltage measurements. Let $\tilde{V}_{i j}=V_{i j}+\varepsilon_{i j}$ and $\tilde{V}_{j k}=V_{j k}+\varepsilon_{j k}$ be two noised voltage measurements with $\varepsilon \sim N(0, \sigma)$, the voltages relation $\tilde{V}_{i k}=\tilde{V}_{i j}+\tilde{V}_{j k}$ implies that the voltage $\tilde{V}_{i k}$ contains a white noise with standard deviation $2 \sigma$. By noticing this noise magnification fact, we suggest applying superposition principle on a smaller number of boundary conditions. In this study, we modify the voltage measurement under adjacent injection pattern (Figure 1(a)). Using the superposition principle we generate a set of hypothetical measurement data corresponding to the skip-3 injection pattern shown in Figure 1(b).

In commercial EIT devices, the voltage measurements employing the current injection electrodes are missed. Using the adjacent pattern, in each current injection, there are only 13 independent voltage measurements that can be obtained. The number of independent measurements is further reduced using the proposed modification procedure. This is because, to form a hypothetic current injection from electrodes $\left(e_{i}, e_{k}\right)$, it is necessary to employ all the current injections between these two electrodes. Within the hypothetical skip-3 pattern, for each current injection, there are only 10 independent voltage measurements that can be obtained.

\subsection{Sensitivity Analysis}

EIT image reconstruction requires solving a combination of the forward and inverse problem. The forward problem simulates the voltage distribution induced by current injection under the FEM framework. With this information, the Jacobian matrix $J$, also called the sensitivity matrix, can be calculated. Intuitively, a row of $J$ represents the response of the voltage changes with respect to small impedance changes of each FEM element. For adjacent and skip-3 injection patterns, the sensitivity of voltage measurement on one electrode pair has been presented in Figure 2. It can be observed that, under the skip-3 pattern, voltage measurements have higher sensitivity to the elements located in the center of domain. 


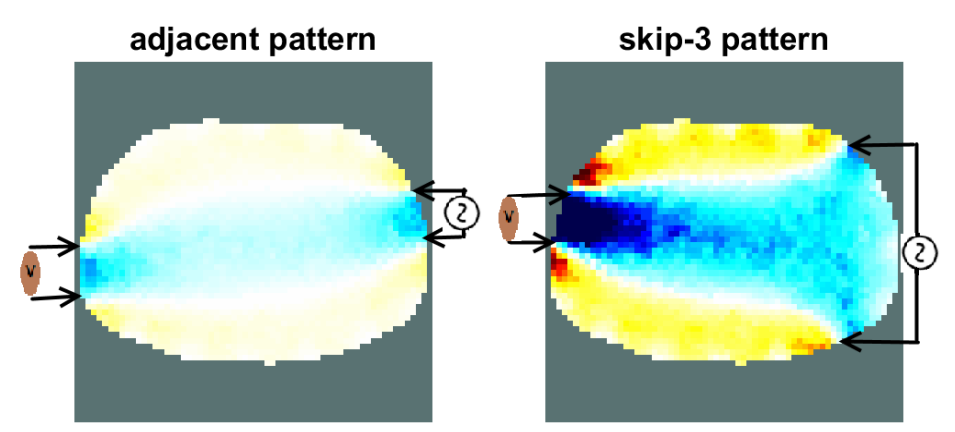

Figure 2. Sensitivity with respect to one of the voltage measurements. The left figure plots the sensitivity of a voltage measurement with respect to each FEM element under the adjacent current injection pattern. The right image showing the same plot regards to the skip- 3 current injection pattern. Both plots are fixed with the same color scale.

(a)

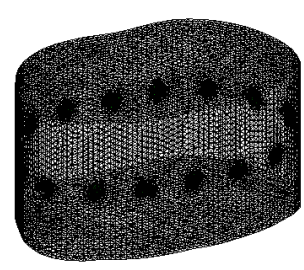

(c)

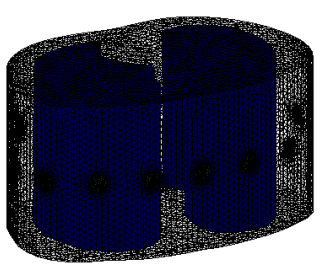

(b)

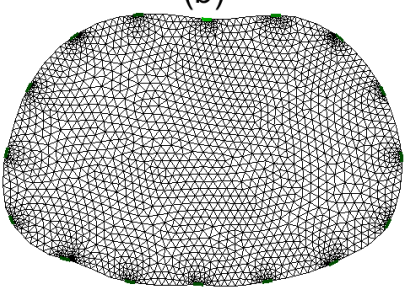

(d)

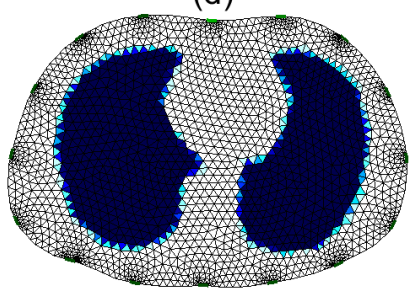

Figure 3. Ground truth for simulation. (a) Is the 3D homogeneous model. Each element has conductivity $1 \mathrm{Sm}^{-1}$. (b) Is a 2D simplification of the 3D model (a). The reconstruction will be performed on this 2D model. The 3D ground truth is displayed in (c). Two contrasts with lung shape are embedded into the homogenous model. These contrasts have conductivity $0.95 \mathrm{Sm}^{-1}$. The 2D simplification of the ground truth on the electrodes level is plotted in (d).

\subsection{Reconstruction with Modified Data}

In this study, we employ the canonical one step Gauss-Newton solver to reconstruct images. Tikhonov regularization was applied to circumvent the ill-posedness of the inverse problem. It is assume that the original voltage measurements are collected with respect to the adjacent current injection pattern. At two time instants, two modified voltages can be obtained according to the method proposed in Section 2.2. Let $\widetilde{\Delta V}$ be a voltage difference between the modified data. As mentioned before, this voltage difference is equivalent to the one resulted from a skip-3 current injection pattern. On the other hand, a Jacobian matrix $J$ with respect to skip-3 current injection pattern will be used for reconstruction. The mathematical formulation of the reconstruction is presented as: 


$$
\widehat{\Delta s}=\arg \min _{\Delta s} \frac{1}{2}\|\widetilde{\Delta V}-J \cdot \Delta s\|_{2}^{2}+\alpha \cdot\|\Delta s\|_{2}^{2}
$$

where $\alpha$ is the Tikhonov regularization parameter. The solution of this problem can be explicitly written by:

$$
\widehat{\Delta s}=\left(J^{T} \cdot J+\alpha \cdot I\right)^{-1} \cdot J^{T} \cdot \widetilde{\Delta V}
$$

\subsection{Simulation Data Acquisition}

The performance of the proposed dada modification method was evaluated through simulation. A 3D simulated homogeneous thorax model was constructed with the help of MATLAB toolbox EIDORS [3]. This model has 50,586 tetrahedron elements. The background conductivity of this homogeneous model was fixed to be $1 \mathrm{Sm}^{-1}$. In this article, all simulated 3D models had a height equal to half of the width. Sixteen electrodes were attached equidistantly around the boundary on the middle level. The contact impedance was fixed to be $100 \Omega$.

Currents of $10 \mathrm{~mA}$ were injected into these electrodes with respect to adjacent as well as skip-3 injection patterns. Corresponding to the skip-3 injection pattern, a Jacobian matrix $J$ can be calculated by the forward solver provided by EIDORS. For reconstruction, the $2.5 \mathrm{D}$ model is employed. That is, the simulation data and Jacobian are calculated on 3D model, but the reconstruction is applied on 2D FEM. This model requires a modification of Jacobian matrix by projecting the Jacobian matrix to $2 \mathrm{D}$ into the electrodes plane. For detail of the 2.5D framework please see [4].

Simulated voltage measurements are calculated using much finer independent 3D FEM models. The voltage measurements are calculated with respect to the adjacent injection pattern. Using a homogeneous model, the boundary voltage $V_{h}$ can be calculated using the forward solver.

Simulated lungs with conductivity $0.95 \mathrm{Sm}^{-1}$ were embedded into an independent homogeneous 3D phantom in order to build an inhomogeneous model (Figure 3(c)). This inhomogeneous model is considered as the 3D ground truth. After embedding the contrasts, another set of voltage measurements under the adjacent injection pattern can be simulated. We denoted this voltage measurement by $V_{i}$. The voltage differences $\delta V=V_{i}-V_{h}$ will be used for reconstruction. Let $N L \in(0,1)$ denote a noise level, a white noise, defined by

$$
\text { Noise }=N L \cdot \operatorname{std}(\delta \mathrm{V}) \cdot \chi
$$

was added to the voltage differences $\delta \mathrm{V}$ for Monte-Carlo simulations. Here std $(\cdot)$ denotes the standard deviation and $\chi \sim \mathcal{N}(0,1)$ is a random vector with Gaussian normal distribution. This noised data will be further modified by the proposed superposition method to form a new set of voltage differences. The original voltage differences and the modified one are both employed in simulation in Section 3 for comparison.

\subsection{Evaluation Parameters}

The relative reconstruction error (RE) of the reconstructed images was evaluated. This 
parameter is defined by:

$$
R E:=\frac{\left\|\Delta s_{s o l}-\Delta s_{g r}\right\|_{2}}{\left\|\Delta s_{g r}\right\|_{2}}
$$

where $\Delta s_{\text {sol }}$ and $\Delta s_{g r}$ represent the 2D reconstructed images and the ground truth respectively.

The system of figure of merit [5] was also employed. These parameters are based on a new image $\widehat{\Delta s_{q}}$, which records the finite elements that have effective conductivity changes. Given a reconstructed image with conductivity changes $\widehat{\Delta s}$, the value of the i-th finite element of $\widehat{\Delta s_{q}}$ is defined by:

$$
\left[\widehat{\Delta s_{q}}\right]_{i}=\left\{\begin{array}{cc}
1 & \text { if abs }[\widehat{\Delta s}]_{i} \geq \frac{1}{5} \max (\operatorname{abs}(\widehat{\Delta s})) \\
0 & \text { otherwise }
\end{array}\right.
$$

In addition, a binary mask on $\Delta s_{g r}$ is defined by:

$$
\left[\Delta s_{C}\right]_{i}=\left\{\begin{array}{cc}
1 & {\left[\Delta s_{g r}\right]_{i} \neq 0} \\
0 & \text { otherwise }
\end{array}\right.
$$

Three figure of merits parameters are defined based on $\widehat{\Delta s_{q}}$ :

Position error: $P E=\left\|r_{t}-r_{q}\right\|_{2}$, where $r_{q}$ and $r_{t}$ are the reconstructed and the original centers of gravity of the contrasts. Position errors of left and right lungs are calculated separately. The sum of these position errors is defined to be the total PE. For this study, we only present the total PE. The position error is expected to be small.

Shape deformation: $S D=\left\|\widehat{\Delta s_{q}}-\Delta s_{C 1}\right\| /\left\|\Delta s_{C}\right\|_{1}$. Shape deformation measures the relative area of misshaping of the reconstruction.

Ring effect: $R N G=\sum_{i \notin C \&[\widehat{\Delta s}]_{i}<0} a b s\left([\widehat{\Delta s}]_{i}\right) / \sum_{i \in C} a b s\left([\widehat{\Delta s}]_{i}\right)$, where $C$ is the true region of the inclusions. The figure of merits parameters are expected to have small variability.

\section{Simulation Results}

Performances of different methods are evaluated by simulation. The simulation model and data were constructed as described in Section 2.4. Four noises levels NL $=0,0.05$, 0.1, 0.2 were used to simulate the measurement noise. One step Gauss-Newton solver (Section 2.3) was employed to reconstruct images. The regularization parameter $\alpha$ used in Equation (5) was determined heuristically to get the best performance. This parameter was set regarding the noise levels. In Figure 4, the reconstructed images with the measurement data with a noise at level 0.05 was demonstrated. Furthermore, the reconstruction error parameters were evaluated in Monte Carlo simulations with 50 independent runs at each noise level. These results are shown in Figure 5.

\section{Discussion and Conclusion}

Several differences between the reconstructed images using original data and the 
(a) With original data

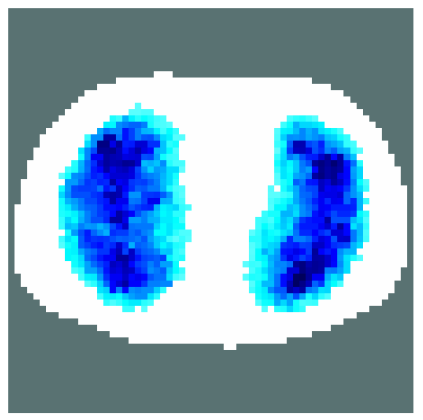

(b) With modified measurment data

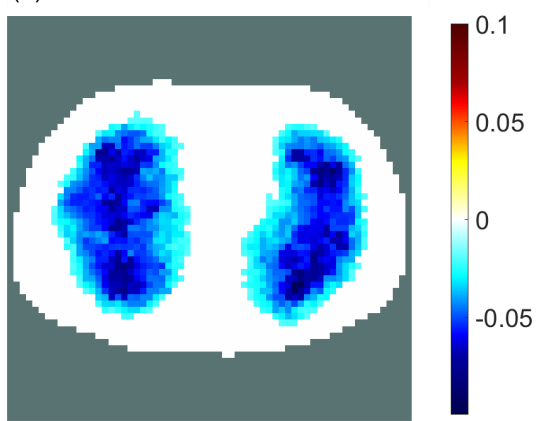

Figure 4. Reconstructed images using original data obtained by adjacent current injection pattern and the modified data proposed in Section 2.3. These reconstructed images are based on the measurement data with a white noise at level 0.05 .
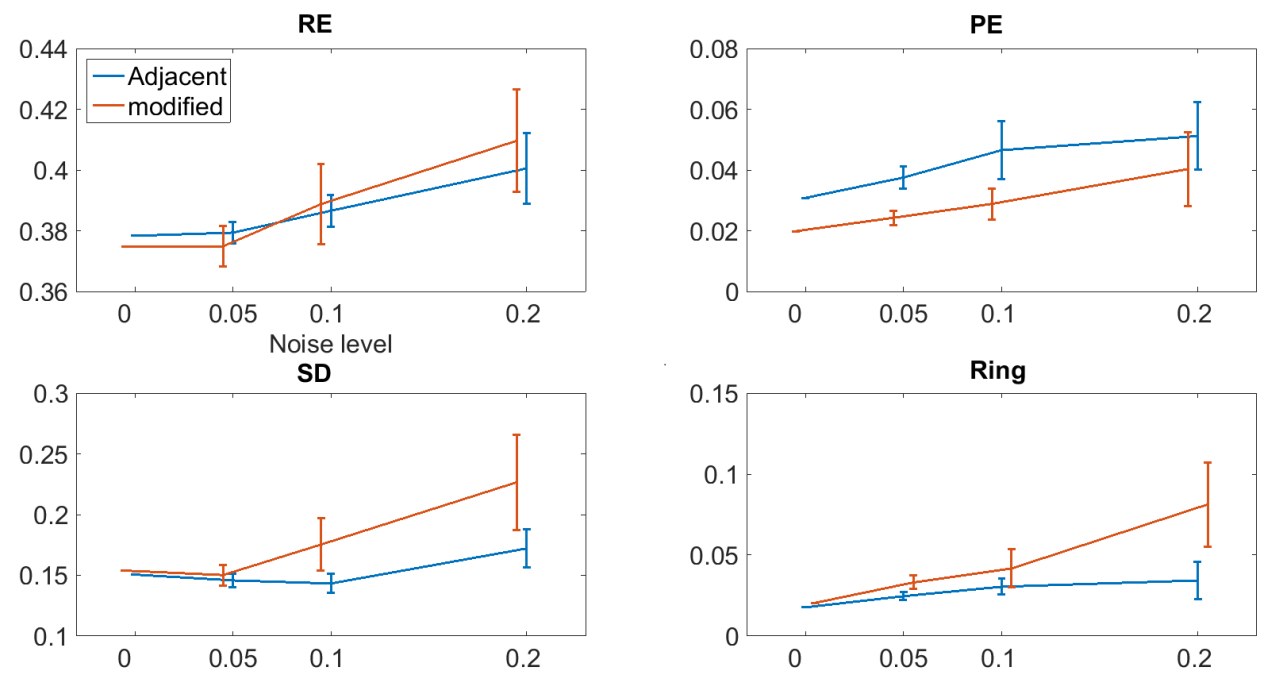

Figure 5. Error analysis based on Monte Carlo simulation. The errors were studied with different noise levels. The image error evaluation parameters were calculated after 50 independent runs of the simulation.

modified data can be observed. The conductivity changes in the central part of the domain are less regarded when using the original data. As comparing the central parts of the two images of Figure 4, it can be observed that reconstructed conductivity changes in the left image are much smaller than the ground truth. As shown by the Monte Carlo simulation results (Figure 5), the position error can be largely corrected by using the modified data.

On the other hand, using original data, the edges of contrasts are better reconstructed around the boundary.

This might be because some of independent measurements are lost in the procedure of modifying data. Based on the same reason, proposed reconstruction method showed more instability. This instability can be read out from the relatively larger variance in the Monte Carlo simulation. To overcome this shortcoming, it might be helpful to complete the missed independent voltage measurements with the ones from original 
voltage data. Consequently, a mixed voltage data set can be constructed. In addition, the Jacobian matrix is required to be modified in the same way to make it consistent with the mixed data.

In this article, EIT imaging using a data modification strategy was studied. The voltage measurements corresponding to adjacent current injection pattern are transformed to those from the skip-3 current injection pattern. Simulation results showed that using the modified data reconstruction is more robust in detecting the central area of the domain, while producing less position error. However, since some independent measurements are lost during data modification, the reconstruction using the modified dada is not stable. To circumvent this difficulty, we suggest completing the modified data with the original data in further studies.

\section{Acknowledgements}

This work is partially supported by the Federal Ministry of Education and Research (BMBF) under grant no. 03FH038I3 (MOSES).

\section{References}

[1] Holder, D.S. (2004) Electrical Impedance Tomography: Methods, History and Applications. CRC Press. http://dx.doi.org/10.1201/9781420034462

[2] Adler, A., Gaggero, P.O. and Maimaitijiang, Y. (2011) Adjacent Stimulation and Measurement Patterns Considered Harmful. Physiological Measurement, 32, 731. http://dx.doi.org/10.1088/0967-3334/32/7/S01

[3] Adler, A. and Lionheart, W.R. (2006) Uses and Abuses of EIDORS: An Extensible Software Base for EIT. Physiological Measurement, 27, S25. http://dx.doi.org/10.1088/0967-3334/27/5/s03

[4] Mamatjan, Y., Borsic, A., Gürsoy, D. and Adler, A. (2013) An Experimental Clinical Evaluation of EIT Imaging with $\ell 1$ Data and Image Norms. Physiological Measurement, 34, 1027. http://dx.doi.org/10.1088/0967-3334/34/9/1027

[5] Adler, A., Arnold, J.H., Bayford, R., Borsic, A., Brown, B., Dixon, P., et al. (2009) GREIT: A Unified Approach to 2D Linear EIT Reconstruction of Lung Images. Physiological Measurement, 30, S35. http://dx.doi.org/10.1088/0967-3334/30/6/s03 
Submit or recommend next manuscript to SCIRP and we will provide best service for you:

Accepting pre-submission inquiries through Email, Facebook, LinkedIn, Twitter, etc.

A wide selection of journals (inclusive of 9 subjects, more than 200 journals)

Providing 24-hour high-quality service

User-friendly online submission system

Fair and swift peer-review system

Efficient typesetting and proofreading procedure

Display of the result of downloads and visits, as well as the number of cited articles

Maximum dissemination of your research work

Submit your manuscript at: http://papersubmission.scirp.org/

Or contact jbise@scirp.org 